\title{
Influencia de padres, amistades y profesorado en la actividad física y la capacidad aeróbica de los jóvenes \\ Influence of parents, friends and teachers on physical activity and aerobic capacity on young people \\ *Martí Xavier Bennàsser Torrandell, **Josep Vidal Conti \\ *Consejería de Educación, Universidad e Investigación de las Islas Baleares (España); **Universidad de las Islas Baleares (España)
}

\begin{abstract}
Resumen: La inactividad física y una baja capacidad aeróbica, implican importantes consecuencias para la salud. El entorno sociopersonal puede influir en la práctica de actividad física (AF) de los jóvenes. El estudio tiene por objetivo determinar qué variables se relacionan con la AF y la capacidad aeróbica de los jóvenes, en función de la influencia de los padres, las amistades y el profesorado. En el estudio participaron 2399 personas de entre 10 y 16 años, de las cuales 1175 fueron chicos (49\%) y 1224 chicas (51\%), con una edad media de 12.7 años. En colaboración con diferentes centros educativos se les administró un cuestionario de hábitos de práctica deportiva en jóvenes, que incluía las siguientes variables: amistades que hacen deporte, percepción de ser animados y ayudados por sus padres y el profesorado a hacer deporte, índice de masa corporal (IMC), volumen máximo de oxígeno (VO2máx.), y horas semanales de AF y de pantalla. Para valorar elVO2máx., se administró el test de course-navette. Para los análisis de los datos se utilizaron pruebas t-student y correlación de Pearson. Los resultados indican, que los jóvenes que tienen amistades que hacen deporte y que perciben ser ayudados por sus padres a hacer deporte, realizan más horas AF semanal, obtienen valores deVO2máx. y de IMC más saludables, y pasan menos tiempo delante de las pantallas. Estos resultados refuerzan la necesidad de establecer estrategias de promoción de la AF, que favorezcan el apoyo familiar y la práctica deportiva entre amistades.
\end{abstract}

Palabras clave: AF, amistades, horas de pantalla, IMC, padres, profesorado, salud, VO2máx.

\begin{abstract}
Physical inactivity and a low aerobic capacity, has significant health consequences. Socio-personal environment can influence on physical activity (PA) of young people. This study aimed to define which variables are related to the PA and the aerobic capacity of young people, based on the influence of parents, friends and teachers. A sample comprised of 2399 young people aged 10 to 16 years, (1175 boys, 49\% - and 1224 girls, 51\%) with a mean age of 12.7 years. In collaboration with different educational centers, a sports habits practice questionnaire in young people was distributed among them, which included the following variables: friends practicing sports regulary, perception of being encouraged and supported by their families, and teachers to do sport, body mass index (BMI), maximum oxygen volume (VO2max.), performed PA hours, and weekly screen hours. The course-navette test, was administered in order to measure the VO2max. T-tests and Pearson's correlation were used for data analysis. The study shows that young people who have friends who doing sports and who perceive that they are encouraged and supported by their parents to do sports, do more PA weekly hours, obtain healthier BMI andVO2max., and spend less time in front of screens. These results reinforce the need to establish strategies to promote $\mathrm{PA}$, which favour family support and sports practice among friends.
\end{abstract}

Keywords: physical activity, friends, screen time, BMI, parents, teachers, health, VO2max.

\section{Introducción}

Los beneficios para la salud de un estilo de vida físicamente activo durante la adolescencia incluyen la mejora de la aptitud cardiorrespiratoria y muscular, la salud ósea y cardiometabólica, y efectos positivos sobre el peso corporal (Physical Activity Guidelines Advisory Committee, 2018).

Para conseguir estos beneficios la Organización

Fecha recepción: 21-02-21. Fecha de aceptación: 20-05-21

Josep Vidal Conti

josep.vidal@uib.es
Mundial de la Salud (OMS), recomienda a la población adolescente, la realización de al menos una media de 60 minutos al día de AF de intensidad moderada a vigorosa; la mayor parte de esa AF debe ser aeróbica (Bull et al., 2020). A nivel mundial el $84.7 \%$ de las chicas y el $77.6 \%$ de los chicos de entre 11 y 17 años, no cumplen con esa recomendación (Guthold, Stevens, Riley \& Bull, 2020). En España, el estudio ANIBES indicó que el 73\% de las chicas de entre 9 y 17 años, no realizaban un mínimo de 60 minutos diarios de AF, mientras que el porcentaje de chicos era del 44,5\% (Mielgo et al., 2016). Además, estos bajos niveles de AF entre las personas jóvenes se han mantenido relativamente estables a lo 
largo de los últimos años (Marques \& Matos, 2014; Guthold, et al., 2020).

Diferentes estudios han relacionado la inactividad Física con valores más reducidos de condición física (Arriscado, Muros, Zabala \& Dalmau, 2015; Rosa et al., 2017). La aptitud cardiorrespiratoria, es considerada como un importante marcador de salud, y se asocia inversamente con factores de riesgo cardiovascular, y de salud mental de los adolescentes (Raghuveer, et al., 2020).

El estudio desarrollado por Tomkinson, Lang \& Tremblay (2019), concluye que a nivel mundial la aptitud cardiorrespiratoria de las personas jóvenes ha ido disminuyendo de forma sustancial desde el año 1981 hasta el año 2000. Entre el año 2000 y 2014 los niveles se han mantenido estables sin cambios significativos. En España, uno de cada cinco jóvenes posee un nivel de aptitud física indicativo de qué en la edad adulta, padecerá alguna enfermedad de índole cardiovascular (hipercolesterolemia, hipertensión, etc.) (Ortega, et al., 2005).

Asociado a la inactividad física y a la baja condición física en los jóvenes, se encuentra el sobrepeso y la obesidad (Ortega, Ruiz \& Castillo, 2013). Un problema de magnitud creciente en muchos países, influyendo considerablemente en la prevalencia de enfermedades no transmisibles y en la salud general, representando el cuarto factor de riesgo de mortandad más importante en todo el mundo (OMS, 2010). Además, la obesidad supone un importante sobrecoste directo en el presupuesto sanitario, con previsiones de un aumento significativo durante los próximos años (Hernáez, et al., 2019). El estudio ALADINO (2019), señala que el porcentaje de sobrepeso infantil en España se sitúa en el $23.3 \%$ y el de obesidad en el $\mathbf{1 7 . 3} \%$.

Otro factor destacable asociado a la inactividad física y a bajos niveles de condición física es el tiempo que pasan los jóvenes delante las pantallas (Lizandra, Devís, Valencia, Tomás \& Peiró, 2019; Cabanas, et al., 2019). El exceso de tiempo de pantalla en jóvenes se asocia negativamente con el desarrollo de capacidades físicas y cognitivas, y positivamente con la obesidad, los problemas de sueño, la depresión y la ansiedad (Domingues, 2017). El Ministerio de Sanidad, Servicios Sociales e Igualdad del gobierno de España, fija la recomendación en un máximo de 2 horas de pantalla diaria (Ministerio de Sanidad, Consumo y Bienestar Social, 2019). Según el reciente estudio desarrollado por Tapia, Sevil \& Sánchez (2021), tan solo el 15.8\% de los adolescentes españoles cumple con esa recomendación.
La práctica de AF y una adecuada condición física se relacionan con la obtención de valores más reducidos de IMC (De Bourdeaudhuij, et al., 2013; Ortega, Ruiz \& Castillo, 2013) y con la disminución de las horas de pantalla (Serrano, et al., 2011; Cabanas, et al., 2019).

Teniendo en cuenta, que los bajos niveles AF y de aptitud cardiorrespiratoria se han mantenido estables durante los últimos años (Marques \& Matos, 2014; Guthold, et al., 2020; Tomkinson, et al., 2019), seria relevante conocer qué factores y en qué grado se pueden relacionar en que una persona joven sea físicamente activa y obtenga valores más saludables de capacidad aeróbica.

Las relaciones sociales ejercen una influencia significativa en la práctica de AF de los jóvenes (Lizarra \& Peiró, 2019). En este sentido las familias representan un importante agente socializador, ya que los niños y las niñas pasan la mayor parte del tiempo en el contexto familiar durante los primeros años de su formación (Lau, Quadrel, \& Hartman, 1990).

Se han desarrollado diferentes estudios centrados en conocer la influencia que ejercen las familias en la práctica de AF de sus hijos e hijas desde diferentes perspectivas; actuando como ejemplo de conducta para el fomento de la AF (Marques, González, Martins, Fernández \& Carreiro, 2017), animando y ayudando en la realización de la AF (Pyper, Harrington \& Manson, 2016; Vaquero, Mirabel, Sánchez \& Iglesias, 2019), o apoyando logísticamente y facilitando la elección de actividades (Welk, Wood \& Morss, 2003). Así mismo otros estudios se han centrado en conocer el efecto de la influencia familiar en el IMC de sus hijos e hijas (Vaquero, Tapia, Cerro \& Sánchez, 2019), y en el tiempo de pantalla (Romero, Dorado, Jiménez, Castro \& Aznar 2020). Estudios previos se han centrado también, en relacionar el nivel de estudios y socieconómico de los padres y las madres, con el nivel de AF de sus hijos e hijas (Cantallops, Ponseti, Vidal, Borràs \& Palou, 2012; Sanchez \& Izquierdo, 2020; Muros, 2021).

Por otro lado, los hallazgos de los estudios observacionales sugieren, que las amistades tienen potencial para afectar la AF y pueden ofrecer oportunidades para promover el desarrollo temprano de hábitos y preferencias saludables de $\mathrm{AF}$, que pueden traducirse en hábitos para toda la vida (Maturo \& Cunningham, 2013). En este sentido los estudios se han centrado en comparar la influencia de las amistades y los factores psicosociales, con la AF y el tiempo frente a las pantallas de los jóvenes (García, Sirard, Deutsch \& Weltman, 2016). También han analizado la influencia de la recipro- 
cidad de la amistad en la AF de los adolescentes. (Schofield, Mummery, Schofield \& Hopkins, 2007).

La Educación Física, como agente relevante en la implementación de programas de promoción de la AF, se convierte en un importante elemento favorecedor de la adherencia hacia la práctica de AF, ya que, al ser obligatoria en el currículum educativo, representa para muchos jóvenes el único lugar donde practican AF regularmente (Sevil, Abós, Generelo, Aibar \& GarcíaGonzález, 2016).

En el mismo sentido el rol del profesorado de Educación Física juega un papel destacado como facilitador y promotor de un estilo de vida activo y saludable, destacando su contribución única en el contexto escolar (Camacho, Fernández, Ramírez \& Blández, 2013). La influencia de dicho profesorado se ha relacionado con la AF extracurricular de los jóvenes (Olivares, Cossio, Gómez, Almonacid \& Garica, 2015), con la adopción de hábitos alimentarios saludables a través de la dieta mediterránea del alumnado (Trigueros et al., 2020), o con la AF desarrollada por los jóvenes desde la perspectiva de género (Bronikowski, et al., 2015).

Hasta el momento no tenemos conocimiento de ningún estudio transversal en jóvenes, que relacione la influencia del entorno sociopersonal con la condición físi$\mathrm{ca}$, y que a su vez se relacione con otras variables como la AF, el IMC y el tiempo de pantalla semanal.

Por todo ello, el objetivo del presente estudio es conocer la influencia del entorno sociopersonal, sobre la AF y la capacidad aeróbica de los jóvenes. Además, se analizará dicha influencia en el IMC y en las horas semanales que pasan los jóvenes delante de las pantallas. Todo ello nos permitiría justificar y diseñar con mayor eficacia programas e intervenciones de promoción de la AF, e incrementar su nivel de participación y de adherencia, evitando factores de riesgo para la salud vinculados al sedentarismo.

Las principales hipótesis que se plantean en el estudio son las siguientes:

Los jóvenes que tienen padres que les animan y ayudan a hacer deporte, realizan más horas de AF semanal, obtienen mayores valores de VO2máx., menores IMC, y pasan menos tiempo delante de las pantallas. Cuanto mayor sea la percepción de la ayuda percibida, realizan más horas semanales de AF, obtienen mayores valores deVO2máx., y pasan menos tiempo delante de las pantallas.

Los jóvenes que tienen amistades que hacen deporte, realizan más horas de AF semanal, obtienen mayores valores de VO2máx., menores IMC, y pasan menos tiempo delante de las pantallas. A mayor número de amistades, realizan más horas semanales de AF, obtienen mayores valores deVO2máx., y pasan menos tiempo delante de las pantallas.

Los jóvenes que actualmente hacen deporte y perciben que las clases de Educación Física les ayuda a que hoy en día practiquen deporte, realizan más horas semanales de AF, y obtienen mayores valores de VO2máx.

\section{Material y Métodos}

\section{Participantes}

Se realizó un estudio de carácter transversal mediante cuestionario, con una muestra representativa de la población de Mallorca (España) de entre 10 y 16 años de edad, como parte del proyecto SAFE (Salud y Actividad Física en la Escuela). La población objeto de estudio fueron escolares de educación secundaria obligatoria (ESO) y quinto y sexto de educación primaria de los colegios de Mallorca. La estimación del tamaño muestral para una población infinita, determinó una muestra teórica de 1066 participantes, con un nivel de confianza del 95\% y un error muestral predeterminado del 3\%. Se envió una carta a todos los centros educativos de Mallorca con la invitación a su participación, y de entre aquellos que aceptaron, se informó por carta a los padres / madres de los participantes, de su carácter voluntario, sobre la naturaleza y el propósito del estudio y se requirió el consentimiento informado por escrito.

La muestra se obtuvo por conglomerados (centros escolares) y muestreo intencional durante el curso 2014/ 2015. El estudio se llevó a cabo con una muestra final de 2399 participantes, (error muestral del 1.6\% y nivel de confianza del 95\%), con una edad media de 12.7 años (DT 1.9).

\section{Instrumentos}

Para la recogida de información se administró el cuestionario «School Health Action, Planning and Evaluation System (SHAPES)» (Wong, Leatherdale \& Manske, 2006), cuyo módulo de actividad física consta de 45 preguntas de opción múltiple, de recuerdo de actividad física moderada a vigorosa (7días), además, informa sobre la participación en actividades físicas, las actividades sedentarias (mirando televisión, jugando videojuegos, tareas), las influencias sociales (compañeros), y el medio ambiente de la escuela para niños de 11 a 16 años de edad. Presenta una fiabilidad alfa de Cronbach de .633 .

Las variables analizadas en el presente estudio fue- 
ron: edad (año de nacimiento); altura (cm); peso $(\mathrm{kg})$; IMC $\left(\mathrm{kg} / \mathrm{m}^{2}\right)$; AF (horas semanales); VO2máx. (ml/ $\mathrm{kg} / \mathrm{min})$; amistades que hacen deporte (1/2/3/4/5); ánimo de los padres hacia la práctica deportiva (me desaniman mucho, me desaniman, no les importa, me animan, me animan mucho); ayuda de los padres hacia la práctica deportiva (no me ayudan nada, no me ayudan, me ayudan, me ayudan mucho); ayuda de la Educación Física hacia la práctica deportiva (si/no) y tiempo de pantalla (horas semanales).

Además, se utilizó la prueba de campo denominada test de Léger, también conocida como test de Course Navette para obtener el VO2máx. (Léger \& Lambert, 1982), ampliamente documentada su validez y fiabilidad (Jódar, 2003).

\section{Procedimiento}

Todos los participantes fueron previamente informados de los objetivos del estudio y su protocolo. El consentimiento informado se envió a cada centro para que fuera entregado a las familias y fue el propio centro quien los recogió. Una vez recogidos se pusieron en contacto con el grupo investigador para proceder a la toma de datos.

Los cuestionarios se administraron por parte de encuestadores formados específicamente para este estudio y con la colaboración de los docentes de los centros educativos. Los cuestionarios fueron distribuidos entre los participantes, adjuntándose las pertinentes instrucciones de uso para su correcta utilización. Durante las clases de educación física se llevó a cabo el test de Course Navette.

Dependiendo de las posibilidades organizativas de cada centro educativo, la administración del cuestionario y la realización del test se realizó el mismo día o en días diferentes. La administración del cuestionario tuvo una duración de unos 20 minutos aproximadamente, y la realización del test de Course Navettte unos 15 minutos como máximo.

El peso y la talla fueron autoreportados por los propios jóvenes y el IMC se obtuvo a partir de aplicar la fórmula: $\operatorname{peso}(\mathrm{kg}) / \operatorname{talla}\left(\mathrm{m}^{2}\right)$.

Este estudio fue aprobado por el Comité de Ética de la Universidad de las Islas Baleares (156CER20).

\section{Análisis estadístico}

Para los análisis descriptivos (tabla 1) se calcularon medias y desviaciones típicas, para las variables continuas y para las categóricas se estimaron frecuencias. La normalidad de las variables fue determinada con la prue- ba de Shapiro-Wilk, y dado que las condiciones se cumplieron $(\mathrm{p}>.05)$, se llevaron a cabo pruebas paramétricas.

Para estudiar la relación entre las variables continuas (horas de AF semanales, VO2máx., amistades activas, padres que animan y apoyan y el IMC) se realizaron análisis de correlación de Pearson (tabla 2), mientras que para la comparación de medias de variables continuas (horas semanales de AF y VO2máx) según la ayuda de la Educación Física, se utilizó la prueba de tstudent (tabla 3).

Todos los análisis fueron realizados mediante el paquete estadístico SPSS-23 para Windows. Para la elaboración de los gráficos se utilizó el programa Excel de Microsoft Office 365.

\section{Resultados}

Los resultados obtenidos a partir de los cuestionarios y pruebas aplicadas, muestran que las personas participantes manifestaron realizar una media de 10.10 (DT 6.90) horas semanales de AF, y obtuvieron un valor medio de VO2 máx. de $34.37 \mathrm{ml} / \mathrm{kg} / \mathrm{min}$. (DT 5.79) y un IMC medio de 19.61 (DT 3.50). Las medidas de IMC determinaron, 238 personas jóvenes con infrapeso (11.54\%), 1268 con normopeso (61.49\%), 434 con sobrepeso (21.05\%) y 122 con obesidad (5.92\%). Así mismo las personas participantes en el estudio manifestaron pasar una media de 21.39 (DT 15.12) horas semanales delante de las pantallas (Tabla 1).

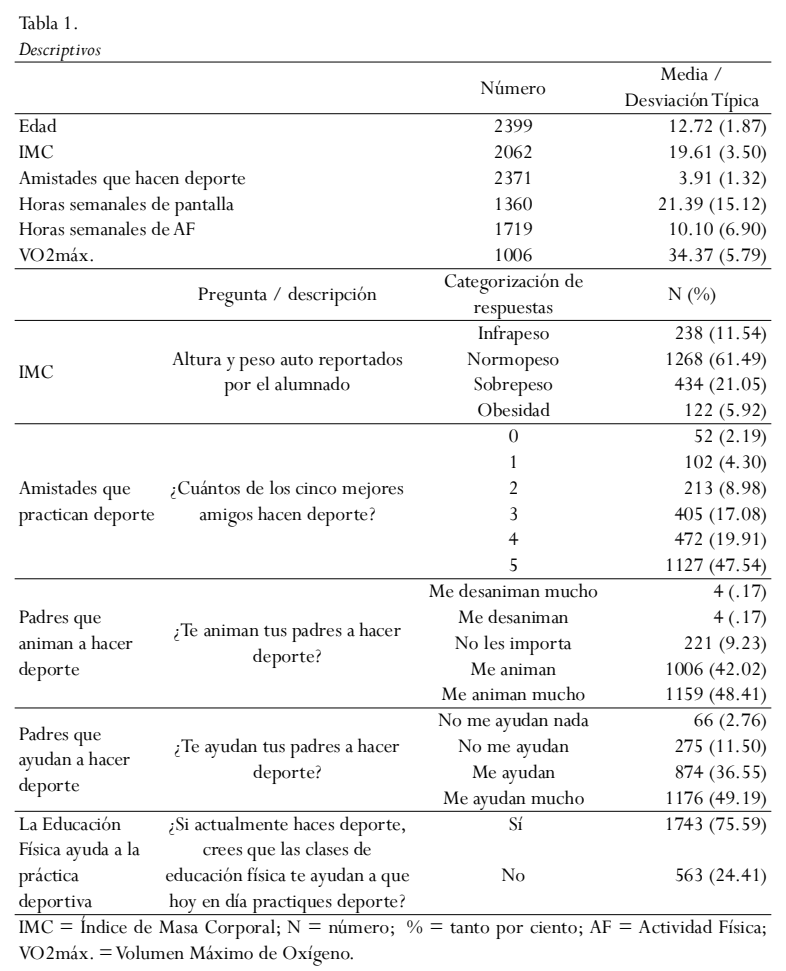


En relación a las amistades que practican deporte, $52(2.19 \%)$ personas jóvenes respondieron que no tenían ninguno de sus cinco mejores amigos que hicieran deporte, 102 (4.30\%) tenían uno, 213 (8.98\%) tenían dos, 405 (17.08\%) tenían tres, 472 (19.91\%) tenían cuatro y 1127 (47.54\%) tenían cinco, con una media total de 3.91 (DT 1.32) (Tabla1). Tener amistades que hacen deporte, se correlaciona con más horas de AF semanal $(r=.209 ; p<.01)$, con mayores valores de VO2 máx. $(\mathrm{r}=.286 ; p<.01)$, con menores valores de IMC $(\mathrm{r}=$ -.076; $p<.01)$, con menos horas de pantalla semanal ( $\mathrm{r}$ $=-.116 ; p<.01)$ y con padres que les animan $(\mathrm{r}=$ $.268 ; p<.01)$ y ayudan $(\mathrm{r}=.335 ; p<.01)$ a que hagan deporte (Tabla 2). Así mismo cuantas más amistades tengan que practiquen deporte, estas personas jóvenes realizan más horas de AF semanal $(\mathrm{t}=18.65$; $p=<$ $.001)$, pasan menos tiempo delante de la pantalla $(\mathrm{t}=$ 4.63; $p=<.001)$ y obtienen mayores valores de VO2 máx. $(\mathrm{t}=19,56 ; p=<.001)($ Figura 1$)$.

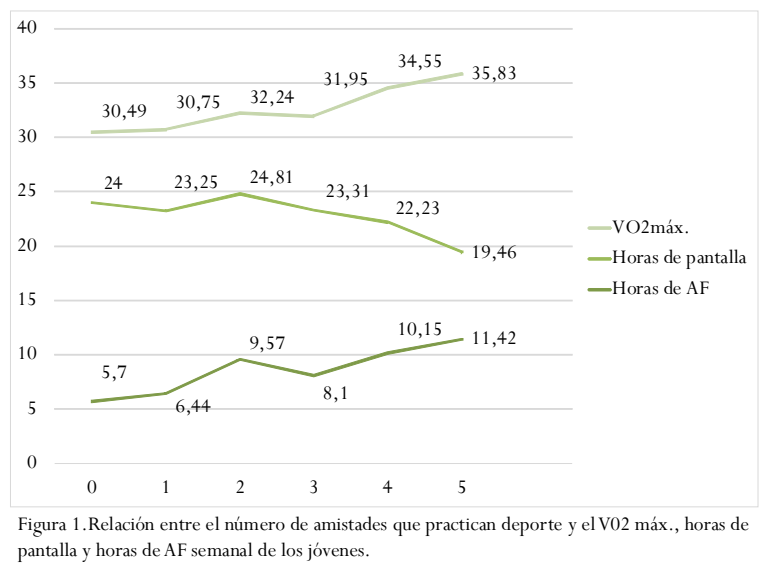

En respuesta a la percepción del ánimo a hacer deporte que reciben las personas jóvenes por parte de sus padres, cuatro (17\%) personas estimaron que les desaniman mucho, cuatro (17\%) que les desaniman, 221 (9.23\%) que no les importa, 1006 (42.02\%) que les animan y 1159 (48.41\%) que les animan mucho (Tabla 1). El ánimo percibido por las personas jóvenes se correlaciona con la realización de más horas de AF semanal $(r=.222 ; p<.01)$, con mayores valores de VO2 máx. $(\mathrm{r}=.216 ; p<.01)$, con menores IMC $(\mathrm{r}=-.075$; $p<.01)$, con menos horas de pantalla semanal $(\mathrm{r}=$ $.113 ; p<.01)$, y con tener más amistades que practican Tabla 2.

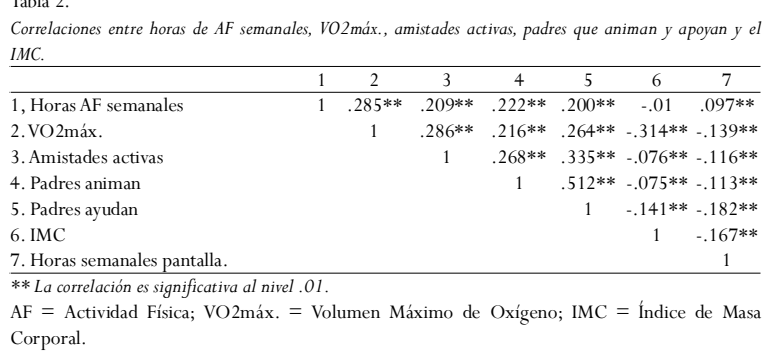

deporte $(r=.268 ; p<.01)($ Tabla 2$)$.

En cuanto a la percepción que tienen las personas jóvenes de ser ayudadas por sus padres; 66 (2.76\%) indicaron no recibir ninguna ayuda, 275 (11.50\%) que no eran ayudados, 874 (36.55\%) que eran ayudados y 1176 (49.19\%) que eran muy ayudados por sus padres (Tabla 1). La ayuda percibida por las personas jóvenes se correlaciona con la realización de más horas de AF semanal $(\mathrm{r}=.200 ; p<.01)$, con mayores valores deVO2 máx. $(\mathrm{r}=.264 ; p<.01)$, con menores valores de IMC $(\mathrm{r}=-.141 ; p<.01)$, con menos horas de pantalla semanal $(\mathrm{r}=-.182 ; p<.01)$ y con tener más amistades que practican deporte $(r=.335 ; p<.01)$ (Tabla 2). Así mismo existe una relación entre cuanto más sean ayudadas por sus padres a hacer deporte, estas personas realizan más horas de AF semanal $(\mathrm{t}=24.70 ; p=<$ $.01)$, pasan menos tiempo delante de la pantalla $(\mathrm{t}=$ $17.71 ; p=<.01)$ y obtienen mayores valores de VO2 máx. $(\mathrm{t}=28.56 ; p=<.001)$ (Figura 2).

Se establece una correlación directa entre el núme-

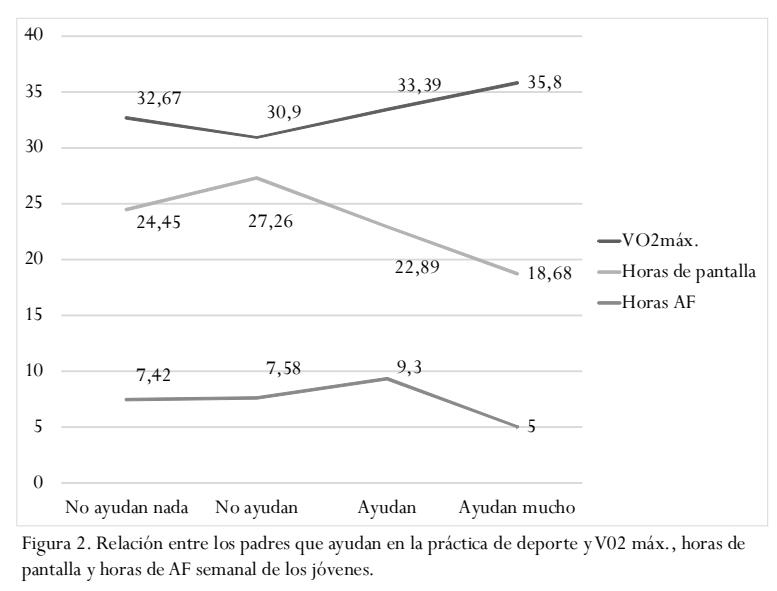

ro de amistades que practican deporte y la ayuda y ánimo de los padres, con la realización de más horas de AF semanal y la obtención de valores más saludables de VO2máx., por parte de los jóvenes. En cambio, se establece una correlación inversa con el IMC y las horas semanales que pasan los jóvenes delante de las pantallas.

La ayuda que ha supuesto la Educación Física hacia la práctica deportiva, en aquellas personas que hoy en día practican deporte; no se relaciona con la realización de más horas semanales de AF $(\mathrm{t}=.69 ; p=<.49)$, ni con mayores valores de VO2 máx. ( $\mathrm{t}=-.79 ; p=<.43)$. (tabla 3)

Tabla 3

\begin{tabular}{|c|c|c|c|c|c|c|}
\hline & $\begin{array}{c}\text { Ayuda de la } \\
\text { Educación Física }\end{array}$ & Número & Media & $\begin{array}{l}\text { Desviación } \\
\text { Típica }\end{array}$ & $\mathrm{t}$ & Sig. \\
\hline \multirow{2}{*}{ Horas semanales AF } & Sí & 1238 & 10.25 & 6.94 & \multirow{2}{*}{.69} & \multirow{2}{*}{.49} \\
\hline & No & 419 & 9.98 & 6.73 & & \\
\hline \multirow{2}{*}{ VO2máx. } & Sí & 746 & 34.4 & 5.7 & \multirow{2}{*}{-.79} & \multirow{2}{*}{.43} \\
\hline & No & 214 & 34.75 & 5.99 & & \\
\hline
\end{tabular}


Los resultados determinan también una correlación entre la realización de más horas de actividad física semanal por parte de las personas jóvenes, con mayores valores de VO2 máx. $(r=.285 ; \mathrm{p}<.01)$, con menores IMC $(r=-.01 ; \mathrm{p}<.01)$ y en pasar más horas semanales delante de las pantallas $(\mathrm{r}=.097 ; \mathrm{p}<.01)$.

La obtención de mayores valores de $\mathrm{VO} 2$ máx., se correlaciona con pasar menos horas semanales delante de las pantallas $(\mathrm{r}=-.139 ; p<.01)$ y con la obtención de menores IMC $(\mathrm{r}=-.314 ; p<.01)$ (Tabla 2).

\section{Discusión}

El objetivo del estudio fue el de determinar qué variables se relacionan con la AF y la capacidad aeróbica de los jóvenes, en función de la influencia de su entorno sociopersonal. Además, se analiza dicha influencia en el IMC y en las horas semanales que pasan los jóvenes delante de las pantallas.

De los resultados se desprende, que la influencia del entorno sociopersonal de los jóvenes de entre 10 y 16 años, se relaciona positivamente con distintas variables estudiadas. A nivel general observamos que estos jóvenes que tienen amistades que hacen deporte, y padres que les animan y ayudan a hacer deporte, realizan más horas de AF semanal, obtienen mayores valores de VO2máx., menores IMC, y pasan menos tiempo delante de las pantallas.

En relación a la primera hipótesis del estudio, los resultados indican una correlación entre los padres que animan y ayudan a sus hijos e hijas a hacer deporte y la realización de más horas de AF semanal $(\mathrm{p}<.01)$, la obtención de valores más altos de VO2máx. $(\mathrm{p}<.01)$, menores IMC $(\mathrm{p}<.01)$ y la permanencia de menos horas semanales delante de las pantallas $(\mathrm{p}<.01)$.

Respecto a la AF, los resultados coinciden con la revisión sistemática desarrollada por Edwardson \& Gorely (2010), y con el estudio transversal realizado por Pyper et al., (2016) donde se destaca la relevancia de diferentes conductas de apoyo de los padres para que los adolescentes sean físicamente activos. En cuanto alVO2máx., los resultados coinciden con los del estudio de López, Marques, Suarez \& de laTorre (2019), donde se concluye que existe una relación positiva entre la capacidad aeróbica y el apoyo parental percibido. En cuanto al IMC, el trabajo llevado a cabo por Vaquero et al., (2017), muestra resultados similares al del presente estudio, estableciendo la importancia que representa el apoyo de los padres en la realización de más horas de AF semanal y en la consecución de valores más reducidos de
IMC por parte de los jóvenes. En relación al tiempo de pantalla, los resultados coinciden con el reciente estudio de Romero et al., (2020), donde se observa que los jóvenes que tienen algún miembro de la familia que hace deporte, pasan menos horas delante de las pantallas y realizan más horas de AF semanal.

Los resultados relacionados con la segunda hipótesis indican una correlación entre las amistades que hacen deporte y la realización por parte de los jóvenes, de más horas de AF semanal $(\mathrm{p}<.01)$, la obtención de valores más altos de VO2máx. $(\mathrm{p}<.01)$, menores IMC $(\mathrm{p}<.01)$ y en pasar menos horas delante de las pantallas $(\mathrm{p}<.01)$.

En cuanto a las horas de AF semanal, los resultados coinciden con la revisión realizada por Maturo \& Cunningham (2013), donde se indica que las amistades tienen potencial para afectar la AF y ofrecen oportunidades para promover el desarrollo temprano de hábitos y preferencias saludables de AF. En el mismo sentido, el estudio de Arévalo, De la Cruz \& Feu (2017) señala que cuando las amistades son más activas, los escolares son más activos y tienen una mayor intención de ser activos en el futuro. Respecto al tiempo de pantalla, los resultados coinciden con los del estudio de García et al., (2016), que establece que las amistades activas influyen en las personas jóvenes para que participen en menos actividades frente a las pantallas.

Las amistades que hacen deporte y la ayuda y el ánimo hacia la práctica deportiva que ejercen los padres, favorecen una mayor práctica de actividad física en los jóvenes. La realización de más horas de actividad física semanal, favorece la obtención de mayores niveles de aptitud cardiorrespiratoria y valores más reducidos de IMC. Así mismo ese mayor número de horas semanales de actividad física repercute en la reducción de las horas que pasan los jóvenes delante de las pantallas.

La tercera hipótesis indica que los jóvenes que actualmente hacen deporte y perciben que las clases de Educación Física les ayuda a que hoy en día practiquen deporte, realizan más horas semanales de AF, y obtienen mayores valores de VO2máx. Cabe señalar que en el presente estudio se ha preguntado sobre la influencia de la Educación Física en la práctica de AF en los adolescentes que ya practican deporte.

La hipótesis planteada no se cumple en relación a horas de AF semanal $(p<$.49), ni en los valores de VO2máx $(\mathrm{p}<.43)$. Los resultados no coinciden con otros estudios, que han analizado la influencia del profesorado de Educación Física en la práctica de AF de los adolescentes, aunque estos estudios han analizado esa 
influencia sin diferenciar si los jóvenes realizaban o no deporte. En este sentido estudios como el de Trigueros, Aguilar, Cangas, López \& Joaquín (2019), destacan la influencia e importancia del profesorado de Educación Física en la adquisición de hábitos de vida activa por parte de los jóvenes.

Esta falta de coincidencia con otros estudios se podría explicar, por qué la práctica deportiva que desarrollan estos jóvenes, pudiera disminuir la percepción de la influencia constante, pero menos directa, que ejercen las clases de educación física sobre la propia práctica deportiva.

Un aspecto destacable del estudio, es la obtención de menores valores de IMC por parte de aquellas personas jóvenes que tienen amistades que hacen deporte $(\mathrm{p}<.01)$ y son animadas y ayudadas por sus padres a hacer deporte $(p<.01)$. Estos resultados son relevantes teniendo en cuenta que hasta donde sabemos, se desconocen estudios que hayan relacionado las amistades que hacen deporte con el IMC de los jóvenes. Además, elevados IMC implican un importante factor de riesgo de enfermedades no transmisibles, como las vasculares, la diabetes, trastornos del aparato locomotor y algunos cánceres (OMS, 2010) y que unos niveles reducidos de IMC en la juventud implicaran niveles reducidos en la edad adulta (Freedman, et al., 2005; Ward, et al., 2017).

De los resultados se desprende una correlación significativa negativa entre las horas de AF semanal y el IMC $(\mathrm{p}<.01)$. Estos resultados coinciden con el estudio de Katzmarzyk et al., (2015), donde se concluye que el desarrollo de AF diaria se asocia con una menor obesidad en una muestra multinacional de jóvenes. En relación también con el IMC y de acuerdo con otros estudios como el de Mitchell, Rodríguez, Schmitz \& Audrain-McGovern (2013), los resultados reflejan una asociación positiva entre el IMC y el tiempo de pantalla.

Por otra parte, los resultados indican que aquellas personas jóvenes que tienen amistades que hacen deporte y que perciben ser animadas y ayudas por sus padres a hacer deporte, obtienen valores más elevados de VO2máx. ( $p<.01)$. Son resultados relevantes teniendo en cuenta que la aptitud cardiovascular es un marcador directo del estado fisiológico, que se asocia inversamente con factores de riesgo cardiovascular y con diferentes enfermedades crónicas (Ortega, Ruiz, Hurtig-Wennlöf \& Sjöström, 2008). Además, el nivel de capacidad aeróbica que se tiene en la juventud se relaciona con factores de riesgo de enfermedad cardiovascular en la edad adulta (Twisk, Kemper \& Van Mechelen 2002).

Los resultados reflejan una correlación significativa positiva entre las horas de AF semanal y elVO2máx. ( $p$ $<$.01). Esta correlación coincide con diferentes estudios que han relacionado la práctica de AF, con mejores niveles de capacidad aeróbica (Arriscado, Muros, Zabala \& Dalmau, 2015; Rosa, et al., 2017).

Cabe mencionar en relación al VO2máx., y al IMC, la existente correlación inversa entre los niveles de VO2máx., y el IMC ( $<<.01)$, en aquellos jóvenes que perciben que son animados y ayudados por sus padres a hacer deporte y que tienen amistades que hacen deporte. Estos resultados coinciden con el estudio de Mondal \& Mishra (2017), que refleja una correlación negativa significativa entre el VO2máx. y el IMC. En cambio, el trabajo desarrollado por Ortega et al., (2008), afirma que las personas adolescentes físicamente activas presentan una mayor probabilidad de tener una capacidad cardiovascular saludable, independientemente de su nivel de adiposidad.

Diferentes estudios han analizado factores relacionados con elVO2máx. de los jóvenes, pero hasta donde sabemos ninguno había incluido la influencia de las amistades y el profesorado, como posible variable asociada. Es por este motivo que creemos que se abre una nueva ventana a explorar y que se requiere de un estudio de mayor profundidad al respecto.

La principal limitación de este estudio es el escaso porcentaje de jóvenes que manifestaron no tener amistades que hicieran deporte y no recibir ni ayuda ni ánimo por parte de los padres a hacer deporte. Otra limitación es que el IMC y la AF semanal fue auto reportada. En el caso del IMC los datos se obtuvieron a través del peso y talla que declararon los jóvenes, y aunque podría ser más adecuado haber sido pesados y tallados de forma objetiva, estudios como los de Galán, Gandarillas, Febrel \& Meseguer (2001) y Sevane et al., (2013), muestran que su análisis como variable continua en jóvenes, conlleva un escaso margen de error.

Entre las fortalezas de la investigación cabe mencionar, que es un estudio representativo de la población de Mallorca con una amplia muestra de 2.399 participantes. Otra fortaleza destacable es la obtención de los valores de VO2máx. de forma indirecta a través de pruebas de campo.

En conclusión, el estudio pone de manifiesto que los jóvenes que tienen amistades que hacen deporte y que perciben ser animados y ayudados por sus padres a hacer deporte, realizan más horas AF semanal, obtienen 
mayores valores de VO2máx. y menores IMC, y pasan menos tiempo delante de las pantallas. Además, el aumento de la percepción de la ayuda familiar y del número de amistades que realizan deporte, favorece que los jóvenes realicen más horas de AF, obtengan mayores valores de VO2máx. y pasen menos tiempo delante de las pantallas. Se evidencia pues, la necesidad de enfatizar en la importancia de que las familias animen y ayuden a sus hijos e hijas hacia la práctica deportiva, y de establecer estrategias de práctica deportiva entre los grupos de amistades.

Para futuras investigaciones resultaría útil estudiar la relación de causalidad y tener en cuenta otras variables como la edad y el género.

\section{Referencias}

Agencia Española de Consumo, Seguridad Alimentaria y Nutrición. Estudio ALADINO 2019: Estudio de Vigilancia del Crecimiento, Alimentación, AF, Desarrollo Infantil y Obesidad en España 2019. Agencia Española de Consumo, Seguridad Alimentaria y Nutrición. Ministerio de Sanidad, Servicios Sociales e Igualdad. Madrid, 2020.

Arévalo, F., De la Cruz, D., \& Feu, S. (2017). La influencia de los padres e iguales en la realización de actividad físicodeportiva de los escolares de educación primaria. Ebalonmano.com: Revista de Ciencias del Deporte, 13(3), 263272.

Argiropoulou, E. C., Michalopoulou, M., Aggloussis, N., \& Avgerinos, A. (2004). Validity and Reliability of Physical Activity Measures in Greek High School Age Children. Journal of Sports Science and Medicine. 3, 147-159.

Arriscado, D., Muros, J. J., Zabala, M., \& Dalmau, J. M. (2015). Hábitos de práctica física en escolares: factores influyentes y relaciones con la condición física. Nutrición Hospitalaria, 31(3), 1232-1239. doi: 10.3305/ nh.2015.31.3.8186

Bronikowski, M., Bronikowska, M., Laudañska, I., Kantanista, A., Morina, B., \& Vehapi, S. (2015). PE teacher and classmate support in level of physical activity: The role of sex and BMI status in adolescents from Kosovo. Biomed. Res. Int. 2015, 1-8. doi: 10.1155/2015/290349

Bull, F. C., Al-Ansari, S. S., Biddle, S., Borodulin, K., Buman, M.P., Cardon, G., Carty, C., Chaput, J. P., Chastin, S., Chou, R., Dempsey. P. C., Dipietro, L., Ekelund. U., Firth, J., Friedenreich, C. M., Garcia, L., Gichu, M., Jago, R., Katzmaryk, P.T., ... Willumsen, J. F. (2020). World Health Organitation 2020 guidelines on physical activity and sedentary behavior. British Journal of Sports Medecine, 54(24), 1451-1462. doi: 10.1136/bjsports-2020-102955

Cabanas, V., Martínez, D., Esteban, I., Pérez, A., Castro, J., \& Veiga, O. L. (2019). Associations of total sedentary time, screen time and non-screen sedentary time with adiposity and physical fitness in youth: The mediating effect of physical activity. Journal of Sports Sciences, 37(8), 839-849. doi: $10.1080 / 02640414.2018 .1530058$

Camacho, M. J., Fernández, E., Ramírez, E., \& Blández, J. (2013). La Educación Física escolar en la promoción de la AF orientada a la salud en la adolescencia: una revisión sistemática de programas de intervención. Revista Complutense de Educación, 24(1), 9-26. doi: 10.5209/ rev_RCED.2013.v24.n1.41189

Cantallops, J., Ponseti, F. J.,Vidal, J., Borràs, P.A., \& Palou, P. (2012). Adolescencia, sedentarismo y sobrepeso: análisis en función de variables sociopersonales de los padres y del tipo de deporte practicado por los hijos. Retos, 21, 58. doi: 10.47197/retos.v0i21.34595

De Bourdeaudhuij, I., Verloigne, M., Maes, L., Van Lippevelde, W., Chinapaw, M. J. M., Te Velde, S. J. \& Brug, J. (2013). Associations of physical activity and sedentary time with weight and weight status among 10- to 12-year-old boys and girls in Europe: a cluster analysis within the energy project. Pediatric Obesity, 8(5), 367-375. doi: 10.1111/ j. 2047-6310.2012.00117.x

Domingues, S. (2017). Clinical and psychological effects of excessive screen time on children. In. Journal of Paediatrics and Child Health, 53(4), 333-338. doi.org/10,1111/ jpc. 13462

Edwardson, C. L., \& Gorely, T. (2010). Parental influences on different types and intensities of physical activity in youth: A systematic review. Psychology of Sport and Exercise, 11(6), 522-535. doi: 10.1016/j.psychsport.2010.05.001

Freedman, D. S., Khan, L. K., Serdula, M. K., Dietz,W. H., Srinivassan, S. R., \& Berenson, G. S. (2005). The relation of childhood BMI to adult adiposity: The Bogalusa Heart Study. Pediatrics, 115, 22-7. doi: 10.1542/peds.20040220

Galán, I., Gandarillas,A., Febrel, C., \& Meseguer, C.M. (2001). Validación del peso y la talla autodeclarados en población adolescente. Gaceta Sanitaria, 15, 490-497. doi: 10.1016/ s0213-9111(01)71611-1

García,J. M., Sirard, J. R., Deutsch, N. L. \&Weltman,A. (2016). The influence of friends and psychosocial factors on physical activity and screen time behavior in adolescents: A mixed-methods analysis. Journal of Behavioral Medicine, 39, 610-623. doi: 10.1007/s10865-016-9738-6

Guthold, R., Stevens, G. A., Riley, L. M., \& Bull, F. C. (2020). Global trends in insufficient physical activity among adolescents: a pooled analysis of 298 population-based surveys with 1.6 million participants. The Lancet. Child \& adolescent health, 4(1), 23-35. doi: 10.1016/S23524642(19)30323-2

Hernáez, A., Zomeño, M.D., Dégano, I.R., Pérez-Fernández, S., Goday, A., Vila, J., Civeira, F., Moure, R., \& Marrugat, J. (2019). Exceso de peso en España: situación actual, proyecciones para 2030 y sobrecoste directo estimado para el Sistema Nacional de Salud. Revista Española de 
Cardiologia, 72 (11), 916-924. doi: 10.1016/ j.recesp.2018.07.009

Jódar, R. (2003). Revisión de artículos sobre la validez de la prueba de Course Navette para determinar de manera indirecta el VO2máx. Revista Internacional de Medicina y Ciencias de la AF y el Deporte, 3(11), 173-181.

Katzmarzyk, P.T., Barreira, T.V., Broyles, S.T., Champagne, C. M., Chaput, J-P., Fogelholm, M., \& Church,T. S. (2015). Physical activity, sedentary time, and obesity in an international sample of children. Medicine \& Science in Sports \& Exercise, 47(10), 2062-2069. doi:10.1249/ MSS.0000000000000649

Lau, R., Quadrel, M. J., \& Hartman, K. A. (1990). Development and change of young adults' preventive health beliefs and behaviour: influence of parents and peers. Journal of Health and Social Behavior, 31, 240-259. doi: $10.2307 / 2136890$

Léger, L. A., \& Lambert, J. (1982). A maximal multistage 20$\mathrm{m}$ shuttle run test to predictVO2 max. European Journal of Applied Physiology and Occupational Physiology, 49, 1-12. doi: $10.1007 / \mathrm{bf00428958}$

Lizandra, J., \& Peiró, C. (2019). Las relaciones sociales y su papel en la motivación hacia la práctica de AF en adolescentes: Un enfoque cualitativo. Retos, 37, 41-47. doi: doi.org/10.47197/retos.v37i37.70374

Lizandra, J., Devís, J., Valencia, A., Tomás, J. M., \& Peiró, C. (2019). Screen time and moderate-to-vigorous physical activity changes and displacement in adolescence: A prospective cohort study. European Journal of Sport Science, 19(5), 686-695. doi: 10.1080/17461391.2018.1548649

López, S., Marques, N., Suarez, S., \& de la Torre-Cruz, M. (2019). Análisis preliminar de las relaciones entre el nivel de condición física y el apoyo parental percibido para la práctica deportiva en adolescentes con sobrepeso y obesidad. Retos, 37, 527-531. doi: doi.org/10.47197/ retos.v37i37.71599

Marques, A., \& Matos, M. (2014). Adolescents' physical activity trends over the years: a three-cohort study based on the Health Behaviour in School-aged Children (HBSC). Portuguese survey. Bmj Open, 4(9), e006012. doi: 10.1136/ bmjopen-2014-006012

Marques, A., González, M., Martins, J., Fernández-Villarino, M.A., \& Carreiro da Costa, F. (2017). Relación entre la AF de los adolescentes y la de madres / padres. Revista de Psicología del Deporte, 26(1), 145-156.

Maturo, C. C., \& Cunningham, S. A. (2013). Influence of Friends on Children's Physical Activity: A Review. American Public Health Associaton, 103(27), 23-38. doi: 10.2105/ AJPH.2013.301366

Mielgo, J., Aparicio, R., Castillo, A., Ruiz E., Ávila, J. M., Aranceta, J., Gil,A., Ortega, R. M., Serra, LL., Varela, G., \& González, M. (2016). Physical Activity Patterns of the Spanish Population Are Mostly Determined by Sex and Age: Findings in the ANIBES Study. Plosone, 11(2): e0149969. doi: 10.1371/journal.pone.0149969

Ministerio de Sanidad, Servicios Sociales e Igualdad. AF para la Salud y Reducción del Sedentarismo. Recomendaciones para la población. Estrategia de Promoción de la Salud y Prevención en el SNS. Madrid, 2015.

Ministerio de Sanidad, Consumo y Bienestar Social (2019). Encuesta Nacional de Salud ENSE, España 2017. Serie informes monográficos \#2 -AF, DESCANSOY OCIO. Madrid: Ministerio de Sanidad, Consumo y Bienestar Social.

Mitchell, J. A., Rodriguez, D., Schmitz, K. H., \& AudrainMcGovern, J. (2013). Greater Screen Time is Associated with Adolescent Obesity: A Longitudinal Study of the BMI Distribution from Ages 14 to 18. Obesity, 21, 572575. doi: 10.1002/oby. 20157

Mondal, H., \& Mishra, S. P. (2017). Effect of BMI, Body Fat Percentage and Fat Free Mass on Maximal Oxygen Consumption in Healthy Young Adults. J Clin Diagn Res. Jun;11(6):CC17-CC20. doi: 10.7860/JCDR/2017/ 25465.10039

Muros, J. J. (2021). Adherencia a la Dieta Mediterránea, AF y su relación con el Nivel Socioeconómico en escolares de Primaria de la capital de Granada (The relationship of Mediterranean diet adherence and Physical Activity engagement with Socioeconomic Status. Retos, 41, 485491. doi.org/10.47197/retos.v0i41.86166

Olivares, P. R., Cossio-Bolanos, M. A., Gomez-Campos, R., Almonacid-Fierro, A., \& García-Rubio, J. (2015). Influence of parents and physical education teachers in adolescent physical activity. International Journal of Clinical and Health Psychology, 15(2), 113-120. doi: 10.1016/ j.ijchp.2015.01.002

Organización Mundial de la Salud. (2010). Recomendaciones mundiales sobre AF para salud. Ginebra: Organización Mundial de la Salud.

Ortega, F. B., Ruiz, J. R., Castillo, M. J., Moreno, L. A., González-Gross, M.,Wärnberg, J., Gutiérrez,A., \& Grupo AVENA (2005). Bajo nivel de forma física en los adolescentes españoles. Importancia para la salud cardiovascular futura (Estudio AVENA). Revista española de cardiología, 58(8), 898-909. doi.org/10.1157/ 13078126

Ortega, F., Ruiz, J., Hurtig-Wennlöf, A., \& Sjöström, M. (2008). Physically active adolescents are more likely to have a healthier cardiovascular fitness level independently of their adiposity status. The European youth heart study. Revista espanola de cardiologia, 61(2), 123-9. doi: 10.1016/ S1885-5857(08)60087-0

Ortega, F. B., Ruiz, J. R., Castillo, M. J., \& Sjöström, M. (2008). Physical fitness in childhood and adolescence: A powerful marker of health. Int. J. Obes, 32, 1-11. doi: 10.1038/ sj.ijo.0803774

Ortega, F. B., Ruiz, J. R., \& Castillo, M. J. (2013). Physical activity, physical fitness, and overweight in children and 
adolescents: evidence from epidemiologic studies. Endocrinologia y Nutrición, 60(8): 458- 69. doi: 10.1016/ j.endoen.2013.10.007

Physical Activity Guidelines Advisory Committee, (2018). Physical Activity Guidelines Advisory Committee Scientific Report. US Department of Health and Human Services, Washington, DC.

Pyper, E., Harrington, D., \& Manson, H. (2016). The impact of different types of parental support behaviours on child physical activity, healthy eating, and screen time: a crosssectional study. BMC Public Health 16, 568. doi: 10.1186/ s12889-016-3245-0

Raghuveer, G., Hartz, J., Lubans, D. R., Takken, T., Wiltz, J. L., Mietus-Snyder, M., Perak, A. M., Baker-Smith, C., Pietris, N., \& Edwards, N. M. (2020). Cardiorespiratory Fitness in Youth: An Important Marker of Health: A Scientific Statement From the American Heart Association. Circulation, 142, e101-e118. doi: 10,1161/ CIR.0000000000000866

Rosa, A., García, E., Rodríguez, P. L., Pérez, J. J., Loreto, M., \&Tárraga, P. J. (2017). AF, condición física y calidad de la dieta en escolares de 8 a 12 años. Nutrición Hospitalaria, 34(6), 1292-1298. doi: 10.20960/nh.813.

Romero, C., Dorado, A., Jimenez, F., Castro, N., \& Aznar, S. (2020). School and Family Environment is Positively Associated with Extracurricular Physical Activity Practice among 8-16 years olds. Int. J. Environ. Res. Public Health , 17, 5371. doi: 10.3390/ijerph17155371

Sanchez, A., \& Izquierdo, T. (2020). Factores socioeconómicos que influyen en la salud nutricional y AF de escolares (Influence of socioeconomic factors in the health state of primary education students). Retos, 40, 95-108. doi.org/ $10.47197 /$ retos.v1i40.81106

Savane, F. R., Navarrete, E. M., García de la Hera, M., Giménez, D., González, S., Valera, D., Sempere, M., \& Vioque, J. (2013). Validez del peso y talla auto-referido en población universitaria y factores asociados a las discrepancias entre valores declarados y medidos. Nutrición Hospitalaria, 28, 1633-1638. doi: 10.3305/nh.2013.28.5.6671

Schofield, L., Mummery,W. K., Schofield, G., \& Hopkins, W. (2007). The association of objectively-determined physical activity behavior between adolescent female friends. Res Q Exerc Sport. 78(1): 9-15. doi: 10.1080/ 02701367.2007 .10599398

Sevil, J., Abós, Á., Generelo, E., Aibar, A., \& García-González, L. (2016). Importancia del apoyo a las necesidades psicológicas básicas en la predisposición hacia diferentes contenidos en Educación Física. Retos, 29, 3-8. doi: 10.47197/ retos.v0i29.34855

Tapia, M. A., Sevil, J., \& Sánchez, P. A. (2021) Adherence to 24-Hour Movement Guidelines among Spanish Adolescents: Differences between Boys and Girls. Children 8, 95. doi: doi.org/10.3390/ children8020095

Tomkinson, G. R., Lang, J. J., \& Tremblay, M. S. (2019).Tem- poral trends in the cardiorespiratory fitness of children and adolescents representing 19 high-income and upper middle-income countries between 1981 and 2014. British journal of sports medicine, 53(8), 478-486. doi: 10.1136/ bjsports-2017-097982

Trigueros, R., Aguilar, J. M., Cangas, A. J., López, R., \& Joaquín, F. (2019). Influence of Physical Education Teachers on Motivation, Embarrassment and the Intention of Being Physically Active During Adolescence. Int. J. Environ. Res. Public Health, 16(13), 2295. doi: 10.3390/ ijerph16132295

Trigueros, R., Mínguez, L. A., González, J. J., Aguilar, J. M., Soto, R., Álvarez, J. F., \& Rocamora, P. (2020). Physical Education Classes as a Precursor to the Mediterranean Diet and the Practice of Physical Activity. Nutrients, 12, 239. doi: 10.3390/nu12010239

Twisk, J. W., Kemper, H. C., \& Van Mechelen, W. (2002). Prediction of Cardiovascular Disease Risk Factors Later in Life by Physical Activity and Physical Fitness in Youth: General Comments and Conclusions. Int. J. Sports Med., 23, 44 50. doi: 10.1055s-2002-28454

Vaquero, M., Tapia, M. Á, Cerro, D., \& Sánchez, P. A. (2019). Importancia del rol familiar en la práctica de AF e IMC de escolares adolescentes. Sportis. Scientific Journal of School Sport, Physical Education and Psychomotricity, 5(3), 408-422. doi: 10.17979/sportis.2019.5.3.5463

Vaquero, M., Mirabel, M., Sánchez, P. A., \& Iglesias, D. (2019). AF de padres e hijos adolescentes: un estudio transversal (Physical activity of parents and their adolescents kids: a cross-sectional study). Retos, 37, 505-508. doi: doi.org/ $10.47197 /$ retos.v37i37.71245

Ward, Z. J., Long, M.W., Resch, S. C., Giles, C. M., Cradock, A., L., \& Gortmaker, S. L. (2017). Simulation of growth trajectories of childhood obesity into adulthood. New England Journal of Medicine, 377, 2145-53. doi: 10.1056/ NEJMoa 1703860

Welk, G. J., Wood, K., \& Morss, G. (2003). Parental influences on physical activity in children: an exploration of potential mechanisms. Pediatr Exerc Sci., 15, 19-33. Doi: 10.1123/ pes.15.1.19

Wong, S. L., Leatherdale S.T., \& Manske, S. R. (2006). Reability and validity of a school-Based Physical Activity Questionnaire. Medicine \& Science in Sports \& Exercise. 38, 1593-1600. doi: 10.1249/ $01 . \mathrm{mss} .0000227539 .58916 .35$

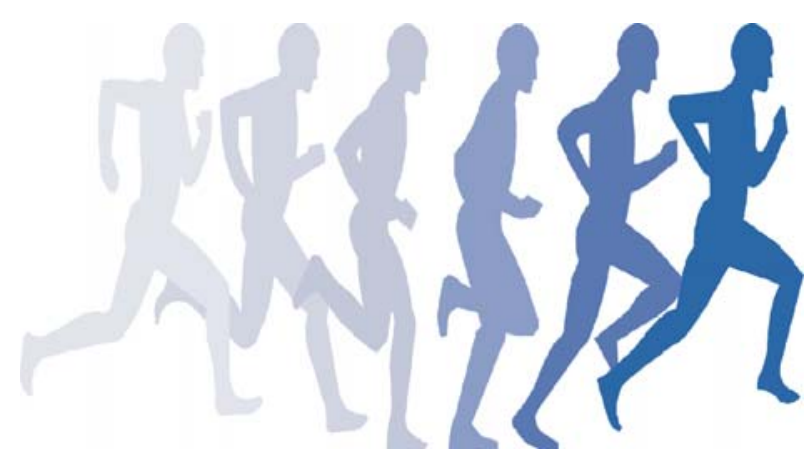

